\title{
The Use of Magnetic Resonance Imaging in Managing Intra-Abdominal Pregnancy
}

\author{
Zainul Ikhwan Ahmad Khusairi\& Tan Ling Sze \\ Emergency and Trauma Department, Taiping Hospital, Perak DarulRidzuan, Malaysia \\ DOI: http://dx.doi.org/10.31344/ijhhs.v5i0.307
}

Introduction: Most pregnant women will experience a normal pregnancy process. However, there are some complications that may occur during pregnancy, including intra-abdominal pregnancy. Intra-abdominal pregnancy, or pregnancy outside of the uterus, are commonly reported besides abortion, pregnancy-induced hypertension $(\mathrm{PIH})$ and gestational diabetes mellitus (GDM). Thesecomplications are believed to be the main cause of maternal and perinatal death. Therefore, the use of appropriate diagnostic imaging inthe management of obstetric cases should be focused.

Objective: This case study intended to evaluate the use of magnetic resonance imaging (MRI) in managing intra-abdominal pregnancy.

Case Presentation: The study focused on one case with intra-abdominal pregnancy which continued into the second trimester. A 30-year-old woman, G2 P0+1 at 12-13 weeks' gestation was presented to a public hospital complaining of recurrent epigastric pain with no history of vaginal bleeding. An MRI was performed and the mother was identified as having an intraabdominal pregnancy. Urgent laparotomy was done. Intraoperative finding noted hematoperitoneum with active bleeding seen from the placenta that adhered to the omentum. Partial omentectomy was done. The foetus in about 14 week's gestation was seen and removed. Case studies have proven that the option to perform an MRI on a suspected intra-abdominal pregnancy can help provide information to establish an accurate diagnosis and appropriate patient care management.

Conclusion: The use of MRI is highly recommended for all cases that are expected to experience intra-abdominal pregnancy as it will save the patient as well as avoiding obstetric complications.

Keywords: magnetic resonance imaging (MRI), intra-abdominal Pregnancy 